\title{
Twelve tips for conducting a virtual OSCE
}

\author{
Abstract \\ Recently many medical schools have faced the challenge of redesigning their existing \\ assessments to run in a virtual format. We ran a virtual Objective Structured Clinical \\ Examination (OSCE) for a group of final year students assessing clinical communication \\ skills, written communication, practical skills, examination skills and professionalism.
} OSCEs provide the opportunity to test skills that written papers cannot, so it was important to include such a clinical exam in the portfolio of assessments for graduating students. The virtual OSCE ran smoothly and was successful at discriminating between candidates. In this article, we share twelve practical tips from our experience and the small body of literature on how to successfully design and deliver a virtual OSCE. This format provides an opportunity to run similar assessments in the future if remote assessments or assessments of telemedicine skills are required.

\section{Introduction}

Objective Structured Clinical Examinations (OSCEs) test skills and attitudes that cannot be examined using written formats of assessment, so are an integral part of the portfolio of medical assessment (Harden and Gleeson 1979). High stakes summative assessments which include a clinical examination component are particularly important for final year medical students to ensure that only competent students graduate and enter the workforce (van der Vleuten 2000).

A small number of postgraduate training programmes and medical schools have completed formative and summative virtual OSCEs. (Palmer et al. 2015; Prettyman et al. 2018; Sartori et al. 2019; Lara et al. 2020). Holding a virtual OSCE has provided the opportunity to assess 
learners who are distance learning or on placement in remote locations (Palmer et al. 2015; Prettyman et al. 2018). Virtual OSCEs have also been used to specifically test telemedicine skills in learners (Sartori et al. 2019); these skills are likely to become increasingly required in routine practice. (Marshall et al. 2020).

In spring and summer 2020, all of our conventional face-to-face assessments were cancelled due to the risks posed to students, examiners and patients by the Covid-19 pandemic. We had several final year students who needed to pass a practical examination in order to graduate and we did not want to delay giving them an opportunity to graduate, so we decided to convene an online eighteen station timed OSCE. Despite this being a virtual OSCE, we preserved the core components of our normal face-to-face OSCE, which meant that students were assessed on clinical communication skills, written communication, practical skills, examination skills and professionalism. The tips below detail some practical advice based on our experience for other institutions who wish to introduce a similar format.

\section{Tip 1: Think about virtual OSCE practicalities early on}

A virtual OSCE is a new concept for most institutions, so you need to spend significant time planning the format to meet your individual needs. This includes considering: the assessment purpose, candidate numbers, which skills you wish to test, stations to include (or exclude), expectations of both staff and students, resources available and the time constraints. Your assessment should satisfy these complex, sometimes conflicting, needs. Ensuring you have information technology (IT) support throughout this process is integral to its success.

Prettyman et al. (2018) describe the importance of collaborating with IT departments for the computer hardware, software, and cloud-based telehealth systems required for the virtual OSCE. 
Another important practicality is how to set-up your OSCE 'circuit'. You may need to run your virtual OSCE in smaller groups than the usual large in-person circuits. For example, Lara et al. (2020) found that they could test just seven students per half day compared with 16 for their face-to-face OSCE. This affects time and human resource management. In our set-up, candidates, administrators (or "hosts"), role players and examiners were together for a three station circuit before the candidate left that online meeting and moved on to the next circuit (of three stations) in another online meeting. This worked well as it reduced the number of different virtual meetings that each student had to join and leave.

\section{Tip 2: Choose a technological set-up that works for your virtual OSCE}

There are multiple online platforms available on which to run your virtual OSCE, including Microsoft Teams, Zoom, Google Hangouts and Adobe Connect. Each platform has pros and cons, and are repeatedly updated with new features, so we would recommend choosing a platform that students, faculty and administrators have most familiarity with. We chose to use Microsoft Teams, as it was already used for teaching sessions and meetings at our institution. We found this worked effectively for audio and visual quality, ease of use and simplicity of displaying information. Lara et al. (2020) and Prettyman et al. (2018) used Zoom effectively. Both authors found the breakout room feature useful as it allowed students to move from the pre-encounter information in the 'waiting room' to a separate virtual 'exam room' where the role player, examiner and an administrator were already positioned.

For formative purposes, it is worth considering software which can easily record stations, giving students the opportunity to watch and learn from their performance (Prettyman et al. 2018). This may also be useful for examiner training and virtual OSCE station development. 


\section{Tip 3: Select your "hosts" carefully}

To make it as simple as possible for students, we chose a set-up that included "hosts". Hosts were administrators whose role was to invite candidates into the stations, ensure they had the correct equipment, display candidate instructions, share their screen if additional documents were required and then time each station. The "hosts" also invited examiners and role players into the stations and were the "friendly face" of the assessment, so that students were put at ease and could perform to their potential. Palmer et al. (2015) and Prettyman et al. (2018) also used this "host" format, where a non-clinical faculty member served as the meeting operator to provide technical support for the session and guide learners through the process. This role of the hosts is relatively difficult due to the many tasks they need to perform but they are integral to the smooth running of the assessment. They need to be calm, organised, familiar with OSCE station requirements, and the platform used whilst remaining welcoming and student focused.

In addition to the "hosts", we found it helpful to have a "superhost". This was a separate, floating member of the team who was available to troubleshoot any problems during the OSCE. Ideally, they should be one of the assessment leadership team who are familiar with all aspects of the assessment and the regulations that support the assessment. They need to be a participant in every online meeting so they are able to discreetly 'drop in' to any station to help solve difficulties and answer any questions that the host or candidates have. This role ensured that the hosts felt supported throughout the assessment and meant that any unforeseen problems were quickly resolved without affecting the performance of the candidates.

\section{Tip 4: Select and modify your stations for an online set-up}


Although you can assess the majority of skills using an online set-up, to help the candidates feel more comfortable, we modified our virtual OSCE stations to establish a more authentic setting without impacting on station focus or delivery. For example, where possible we changed the clinical setting to a remote consultation in general practice. For our practical skills stations we altered our candidate instructions to make it clear that they were doing these skills in a simulated environment, for example in our influenza vaccination station the role player was a GP supervisor asking the candidate "to demonstrate on the orange how you would give the vaccine" before seeing a patient.

\section{Tip 5: Be confident and creative}

We successfully assessed a variety of integrated skills including clinical and written communication, physical examination, interpretation of clinical signs, clinical proficiencies and prescribing. As well as simulated consultations where role players played patients or colleagues, we included media clips of real patients to assess identification and interpretation of clinical signs, and a simulated ward round to assess documentation. We also assessed remote examination skills, such as parts of the neurological exam that only required inspection. We sent students equipment for their clinical and practical skills, including suturing equipment, catheters and equipment for simulated injections. We also asked students to print, in advance, documents to complete written notes and drug charts. With a bit of creativity, it is possible to test almost everything that you normally include in your physical OSCE.

The virtual OSCE format provides the potential to test competence in telemedicine skills specifically, which are likely to become increasingly common in clinical practice. (Marshall et al. 2020). Sartori et al. (2019) found that they were able to implement a series of OSCE 
cases that represented remote encounters, requiring trainees to elicit a history, perform a virtual physical examination, make management decisions and arrange appropriate triage for a standardised patient.

\section{Tip 6: Don't reveal the contents of the stations prior to the OSCE}

As students will need practical equipment for clinical skills and printed documentation, eg hospital prescription charts, for some stations, plan what resources are needed before you release any guidance to the students. You cannot update and release new requirements later, as this will alert students to what you are assessing. For the actual OSCE, students will need to gather all equipment in advance. We recommend that you give students a list of what might be needed for the OSCE, not just the resources they need for the stations you are using. This avoids prompting them to exactly which skills will be tested. For example, they should have all clinical practical skills equipment they were taught with available off-camera so that they cannot predict exactly which clinical skill will come up.

\section{Tip 7: Everyone will need extra training for a virtual OSCE}

Students need a considerable amount of training to ensure that their performance is not affected by the format of assessment and instead reflects their clinical competence. We began well in advance of the OSCE by holding communication skills sessions to help students become familiar with how to perform an online consultation. We provided several opportunities for students to practise online consultations with a role player. Once they are comfortable with online consultations, we recommend you demonstrate the platform and how to use it, for example how to open 'meeting notes' to see their candidate instructions and how 
to 'pin' the role players to ensure they always remain on screen. This should be repeated as many times as is needed, including a couple of days before the assessment.

Students need teaching sessions on demonstrating practical skills, for example suturing, remotely on camera. They should be shown how to find the best area to complete this in their own environment and the best camera angle for the examiner to fully visualise their performance. We would also recommend providing students with opportunities to practise other practical skills such as prescribing and written documentation, so that they are familiar with sending their completed documentation electronically to their examiners for marking.

In addition to training the candidates on the format of the virtual OSCE and the software, it is vital to train the hosts, examiners and role players. Everyone needs to be familiar with their role within the OSCE and logistics of how it will run. It is a new format for everyone, so even if you, as organiser, are confident that it can work, the hosts need to share this confidence so they can pass it on to candidates and role players.

We recommend allocating at least three hours of dedicated time to this training and focusing on increasing confidence with the software format. Holding an initial training session early on, using sample stations, highlights any major problems with technology, equipment or format you may have. A second session close to the assessment keeps things fresh in the minds of the participants.

\section{Tip 8: Keep the focus on the candidate during the assessment}

When hosts and examiners need to look at several pieces of information on a screen there is the potential for them to lose their focus on the candidates. Our examiners needed to review 
marksheets, feedback forms, examiner guidance and the candidate instructions, and our hosts had to review their timing instructions and documents detailing the equipment and role player needed for each station. We would recommend printing hard copies of all these documents so that the hosts and examiners can concentrate on the candidate on their screen.

\section{Tip 9: Optimise what is shown on the candidate's screen}

For each station, the candidate should clearly see their candidate instructions, the role player and any additional documents or media for the station, for example patient notes. The candidate instructions for each station were visible to the candidates at all times using the 'meeting notes' function. This was important so that candidates could refer back to them at any time. If any additional documents were required during a station, the host would share their screen to show this document and candidates were able to take control and expand the image as needed. We would recommend designing all documents in landscape orientation, so the whole document is displayed on one screen which avoids candidates scrolling during a station. We also used only pdf documents so that candidates were comfortable with one consistent format.

Examiners kept their video off throughout the assessment to avoid distracting the candidate. Hosts had their video on initially, to greet and check that candidates could view any required documents or had their prepared equipment ready, then they switched their video off prior to starting the reading time for that station.

\section{Tip 10: Do a full run through (at least once)}

We recommend completing a full run-through of the virtual OSCE before the day. This ensures that everyone is aware of their role and competent at performing it. It allows all 
resources to be checked and tests the communication between the host's computer and the examiner and role players to identify any IT issues. In our case this highlighted, that one host's Apple computer did not show the correct formatting of some documentation when shared with a personal computer (PC). Workarounds are always possible, but such problems need to be identified prior to the exam to be resolved.

During the 'dress rehearsal' set aside enough time to give each examiner and role player an opportunity to enter and leave the station, test their microphone and streaming video, and practice muting and unmuting themselves. The host needs to practise admitting all appropriate people to their online meeting, timings and sharing of any resources. While you do not need your volunteer candidates to complete each stations in full whilst testing the practical aspects of the OSCE, it is helpful to have someone to act in the candidate role and you may wish to use this to pilot the contents of the station. We used our alternative examiner to act as the candidate, as we thought this gave them insight into how it feels to be assessed in the virtual OSCE, which was informative when making their judgements. Lara et al. (2020) completed a full test run with volunteer candidates and found this was helpful to ensure feasibility.

\section{Tip 11: Use an external communication channel for private mid-OSCE communication}

We found it was helpful to have a dedicated external channel for communication between organisers, ensuring that the actual OSCE was not disturbed and the candidates were not included in this communication. We used a separate phone-based text message tool, eg WhatsApp, which allowed rapid communication between different members of the team involved in the OSCE. 
We had one group for the hosts, 'superhost' and OSCE organisers used to co-ordinate moving the candidates between each circuit of three stations, sharing information between hosts and answering any questions. It was not used to share any of the OSCE material.

Candidates also used WhatsApp to send their host any photos of complicated practical work, for example completed prescription charts or sutured bananas. Sharing the host's phone number with the candidate meant that they could contact the host directly in case of connectivity issues (although none arose during our OSCE).

Each circuit also had its own WhatsApp group composed of the host, examiner, and role players. This was used to share any photos that candidates had sent with examiners and for role players to give their scores and feedback to examiners without candidates hearing.

\section{TIP 12: Get detailed feedback from all involved and learn from it for next time}

We obtained feedback from the candidates, hosts, role players and examiners prior to the results becoming available. We specifically asked about face and content validity, acceptability. All involved provided feedback on their experiences. 94\% of those involved (including all students) recommended that a virtual OSCE be offered again should remote assessment be required.

All respondents felt that the virtual OSCE was best for assessing communication skills; however it was also felt to be valid in assessing a range of other skills that would usually be tested face-to-face. This included professionalism and fitness to practise, prescribing, written documentation, integration of skills across several domains in one station, and examination of 
practical skills. The main limitation was its perceived limited ability to assess students' competence in performing physical examinations.

We will use this feedback to design and develop future virtual OSCEs, inform the authors of new virtual OSCE stations and to help alleviate the concerns of students and staff about the use of virtual OCSEs.

\section{Conclusions}

Running a virtual OSCE is achievable and provides an opportunity to test a wide range of clinical skills in a remote setting. It requires planning, additional training and piloting to ensure that all involved are aware of their roles and familiar with the format and software. This change in established practice provides opportunities for clinical skills assessment during this pandemic situation, future assessment of students on remote placements and for the assessment of telemedicine skills.

\section{Acknowledgements}

We would like to acknowledge Paula Raftery, who was our 'superhost', for her commitment and dedication to the organisation of the virtual OSCE.

We would like to thank our hosts, examiners, role players and candidates for their enthusiasm in taking part in this novel format for assessment.

\section{References}

Harden R, Gleeson F. 1979. Assessment of clinical competence using an objective structured clinical examination (OSCE). Med Educ. 13(1):41-54. 
Marshall M, Howe A, Howsam G, Mulholland M, Leach J. 2020. COVID-19: a danger and an opportunity for the future of general practice. Brit J Gen Pract. 70(695):270-271.

Lara S, Foster C, Hawks M, Montgomery M. 2020. Remote assessment of clinical skills during COVID-19: a virtual, high-stakes, summative pediatric OSCE. Acad Pediatr. [accessed 2020 July 1]:[DOI: https://doi.org/10/1016/j.acap.2020.06.002]

Palmer RT, Biagioli FE, Mujcic J, Schneider BN, Spires L, Dodson LG. 2015. The feasibility and acceptability of administering a telemedicine objective structured clinical exam as a solution for providing equivalent education to remote and rural learners. Rural Remote Health. 15(4):3399.

Prettyman AV, Knight EP, Allison TE. 2018. Objective structured clinical examination from virtually anywhere. JNP- J Nurse Pract. 14(8):e157-e163

Sartori DJ, Olsen S, Weinshel E, Zabar SR. 2019. Preparing trainees for telemedicine: a virtual OSCE pilot. Med Educ. 53(5):517-518.

van der Vleuten C. 2000. Validity of final examinations in undergraduate medical training. BMJ Brit Med J. 321:1217-1219. 